\title{
Sensory integration intervention and the development of the premature infant: A controlled trial
}

\author{
E Lecuona, ${ }^{1} \mathrm{M}$ (Occupational Therapy); A van Jaarsveld, ${ }^{2} \mathrm{M}$ (Occupational Therapy); J Raubenheimer, ${ }^{3} \mathrm{PhD}$; \\ R van Heerden, ${ }^{4} \mathrm{M}$ (Occupational Therapy), $\mathrm{PhD}$ \\ ${ }^{1}$ Department of Occupational Therapy, Universitas Academic Hospital, Bloemfontein, South Africa (current affiliation: occupational therapy \\ private practice, Bebe Developmental Care, Cape Town, South Africa) \\ ${ }^{2}$ Department of Occupational Therapy, Faculty of Health Sciences, University of the Free State, Bloemfontein, South Africa \\ ${ }^{3}$ Department of Biostatistics, Faculty of Health Sciences, University of the Free State, Bloemfontein, South Africa (current affiliation: Clinical \\ Pharmacology and Toxicology Research Group, Discipline of Pharmacology, Sydney Medical School, University of Sydney, Australia) \\ ${ }^{4}$ Occupational therapy private practice, Bloemfontein, South Africa
}

Corresponding author: E Lecuona (elise@bebedevelopmentalcare.co.za)

Background. Premature infants are at risk of sensory processing difficulties and developmental delays due to an immature central nervous system and possible episodes of medical instability, discomfort, pain and stress during the first weeks or months after birth.

Objective. To investigate the effect of Ayres Sensory Integration (ASI) on the development of premature infants in the first 12 months of life. Methods. A pre-/post-test experimental design was used to randomly divide 24 premature infants from a low socioeconomic setting in Bloemfontein, South Africa, into experimental and control groups after being matched by corrected age and gender. Developmental status was determined with the Bayley III Scales of Infant and Toddler Development, the Test of Sensory Functions in Infants and the Infant/ Toddler Sensory Profile. The experimental group received 10 weeks of ASI intervention.

Results. ASI intervention had a positive effect on the sensory processing and development of premature infants, especially in terms of cognitive, language and motor development.

Conclusions. ASI intervention at an early age enhances the developmental progress of premature infants.

S Afr Med J 2017;107(11):976-982. DOI:10.7196/SAMJ.2017.v107i11.12393

Small-for-gestational-age and extremely-low-birth-weight (ELBW) premature infants are at increased risk of developmental and cognitive delays, and difficulties in the mother-infant relationship. ${ }^{[1]}$ These infants not only face survival and developmental challenges, ${ }^{[2]}$ but are also at risk of developing sensory integration (SI) difficulties. Their immature, disorganised nervous systems are not ready to process the sensory information bombardment of neonatal intensive care units (NICUs), ${ }^{[2]}$ and they are therefore likely to develop at a slower rate than full-term infants.

Although advances in perinatal care have improved premature infant survival rates, these infants still experience more neurosensory impairments than full-term infants. ${ }^{[3]}$ The progression of normal SI functions during infancy is essential for optimal development, since most activities in the first 7 years of life are part of the process of organising sensations in the nervous system for adaptive responses. Research indicates that during the first 1000 days of life (from conception to age 3), children's brains can form 1000 neural connections per second. A critical window of opportunity is therefore available, and appropriate stimulation from the earliest possible moment could improve cognitive capacity and the chance to live a fuller, more productive life. ${ }^{[4]}$ Sensorimotor organisation occurs through adaptive responses, e.g. babies hear a sound and turn their heads towards the sound. Every child has an inherent drive towards engaging in sensory experiences that will promote SI. The relationship of SI to engagement in daily occupations is well described in the literature, and enables cognitive, motor, social, emotional and body scheme development. ${ }^{[5,6]}$

Ayres $^{[5]}$ explained the SI process as four levels that link the different sensory systems (vestibular, proprioceptive, tactile, auditory and visual) to very specific SI abilities and functions that allow for purposeful participation in activities. The end products of SI include academic learning, self-esteem, self-control and self-confidence. ${ }^{[5]}$

Infants manage behavioural reactions to sensation according to their sensory thresholds through the process of sensory modulation. ${ }^{[7,8]}$ The 'four As' of infancy ${ }^{[8]}$ describe the primary ways in which infants and toddlers perceive and modulate sensory information: arousal (maintaining alertness and making transitions between states); attention (focusing selectively on a desired stimulus or task); affect (emotional component of behaviour); and action (engaging in adaptive, goal-directed behaviour).

Self-regulation activities include finger or dummy sucking, finger playing, self-rocking and looking at or listening to preferred visual or auditory stimuli. These activities enable infants to modulate mood, self-calm, delay gratification and tolerate transitions in activities. Infants with regulatory disorders, however, are unable to use these methods to calm themselves and require extreme efforts by their mothers to calm them. These infants may seem fussy and irritable, and transition quickly from a pleasant mood to an intense cry. Difficulties experienced with self-regulation may negatively affect the development of cognition, language, skilled movement, behaviour and emotional control, and also sensorimotor modulation up to the age of 3 years. ${ }^{[9]}$

Through discrimination of sensory information followed by perception thereof, the infant interprets the qualities of the sensory information and adds meaning to it. Infants have to use past experiences and memories, form associations about the spatial and/ or temporal qualities of what they are currently experiencing, and then act on that. Refined use occurs once integration of sensory information contributes to skills and praxis. ${ }^{[5,10-12]}$ 
Ayres Sensory Integration (ASI) intervention techniques are integrated within the context of parent guidance and child-centred activity, to normalise the child's responses to sensory experiences, modulate arousal and promote organised adaptive responses during play and everyday activities. Rigid treatment protocols are incompatible with the ASI intervention approach. ${ }^{[6]}$

South Africa (SA) has an annual premature birth rate of $>8 \%$. ${ }^{[13]}$ In addition to prematurity-related developmental difficulties and the long-term implications thereof, toddlers from low socioeconomic environments are more likely than those from higher socioeconomic environments to experience difficulties with developmental components dependent on sufficient SI, namely postural control, bilateral motor co-ordination and reflex integration. ${ }^{[14]}$ Research focused on the effectiveness of ASI intervention on the development of premature infants in developing countries such as SA is therefore vital.

\section{Objective}

To investigate the effect of ASI intervention on the developmental progress of ELBW to very-low-birth-weight premature infants from low socioeconomic settings, aged $\leq 12$ months.

\section{Methods}

\section{Research design}

Following a pre-test/post-test experimental design, infants were selected randomly according to their corrected ages and gender into experimental (EG) and control (CG) groups for assessment and intervention purposes.

\section{Sample}

Twenty-four premature infants met the criteria for inclusion (medically stable, birth weight 750 - 1499 g, gestational age 26 - 36 weeks, corrected age 4 - 10 months) and exclusion (no previous occupational therapy or SI intervention and no additional conditions/ neurological abnormalities) and participated in the study. All the infants involved in the study were born in a single neonatal unit. The unit implemented neurodevelopmental supportive care principles that include adapting the NICU environment in terms of lighting, sound, and handling and positioning techniques that support positive growth and development.

\section{Research process: Assessment and intervention}

Medical and background information was obtained from hospital files and parent interviews. An occupational therapist (OT) experienced and trained in the use of the different measuring instruments conducted the pre- and post-test procedures and recorded the data. This therapist was blinded to the infants' group membership. Three standardised measuring instruments were used in this study: the Infant/Toddler Sensory Profile (ITSP), the Test of Sensory Function in Infants (TSFI) and the Bayley III Scales of Infant and Toddler Development (BIII).

The EG was exposed to 10 weekly 45-minute ASI intervention sessions following the pre-tests. A maximum of two appropriate play/handling recommendations were demonstrated to parents after each session. The model of infant behaviour based on SI and self-regulation ${ }^{[15]}$ provided a framework within which intervention planning was organised in terms of: (i) helping parents to understand their infant and his/her developmental progress; (ii) facilitating goodness of fit between infants and their sensory environment; and (iii) addressing the underlying sensory processing and self-regulation problems and/or their behavioural expression in terms of arousal, attention, affect and action.

\section{Data analysis}

Categorical comparisons between the two groups were made by a biostatistician using $\chi^{2}$ analysis with Fisher's exact text (owing to the small sample size). Comparisons between the two groups for scale measurements were made using Student's $t$-test. Data analysis used SAS/STAT software, version 12.3 of the SAS System for Windows (SAS Institute, USA).

\section{Validity and reliability}

All three assessment tools have acceptable validity and reliability. ${ }^{[7,16,17]}$ Although these tests were developed in the USA, they were considered the most suitable for the purpose of this research.

Evidence-based practice is critical in terms of investigating the effectiveness of ASI, so the use of a fidelity measure is essential in ASI research. ${ }^{[6]}$ Ten randomly selected intervention sessions (from the collective 120 EG sessions) were evaluated according to the ASI fidelity measure ${ }^{[6]}$ after completion of the study. Two OTs trained in the use of the ASI fidelity measure rated the intervention sessions independently, and the average of each session was then calculated. The results of the fidelity measure indicated high credibility (mean rating of $85.1 \%$ ) of the use of ASI intervention in all the rated sessions.

\section{Ethical approval}

Ethical approval was obtained from all relevant authorities, including the Ethics Committee of the Faculty of Health Sciences, University of the Free State (ref. no. 117/2011) and appropriate authorities. Informed consent was obtained from the participating infants' parents.

\section{Results}

\section{Demographic and anthropometric data}

There were no statistically significant differences between the two groups, barring the hospitalisation period (in favour of the CG). All 24 participants lived in low socioeconomic settings. The randomisation of the EG and CG successfully resulted in intergroup comparability with regard to the variables shown in Table 1 .

\section{Self-soothing methods}

At post-test, all EG infants used the provided soft taglet (comforting blanket) with positive feedback from parents, and had a wider variety of appropriate self-soothing methods than the infants in the CG (Fisher's exact test $p=0.09$ ): both finger and dummy (pacifier) sucking (EG 41.7\%, CG 0); only dummy sucking (EG 25.0\%, CG 41.7\%); only finger sucking (EG 16.7\%, CG 25.0\%); no finger or dummy sucking (EG 16.7\%, CG 33.3\%).

\section{Test results}

\section{The Infant/Toddler Sensory Profile}

The ITSP evaluates the possible contributions of sensory processing to the infant's daily performance patterns, e.g. eating, play and interaction. Behavioural responses or self-regulation are described as the way infants act in consideration of their neurological thresholds. ${ }^{[7]}$ Literature reports emotional regulation as relying upon prefrontal control of limbic regions, and the anterior cingulate cortex is specified as a key region for the regulation of emotion, ${ }^{[18]}$ enabling participation in functional behavioural outcomes such as state of regulation.

Fig. 1 depicts the behaviour of infants in terms of typical performance, probable differences less, and more, than others, and definite differences more than others (no definite differences less than others were found). 
Table 1. Comparison of infants in the control and experimental groups

\begin{tabular}{|c|c|c|c|}
\hline \multirow[b]{2}{*}{ Variable } & \multicolumn{2}{|c|}{ Group } & \multirow[b]{2}{*}{ Comparison } \\
\hline & $\begin{array}{l}\text { Experimental } \\
(n=12)\end{array}$ & $\begin{array}{l}\text { Control } \\
(n=12)\end{array}$ & \\
\hline Median corrected age (months, days) & 6,21 & 6,12 & $t=0.08, p=0.94$ \\
\hline Mean gestational age (weeks) & 30.3 & 30.7 & $t=-0.41, p=0.69$ \\
\hline Gender, \% & & & $\chi^{2}=0.69, p=0.41$ \\
\hline Girls & 33.3 & 50.0 & \\
\hline Boys & 66.7 & 50.0 & \\
\hline Mean birth weight (g) & 1098 & 1204 & $t=-1.13, p=0.27$ \\
\hline Reason for premature birth, \% & & & $\chi^{2}=7.1, p=0.13$ \\
\hline Pre-eclampsia & 66.7 & 75.0 & \\
\hline Other & 33.3 & 25.0 & \\
\hline Self-soothing methods, \% & & & $\chi^{2}=0.4, p=0.94$ \\
\hline Finger sucking & 58.3 & 50.0 & \\
\hline Dummy & 8.3 & 16.7 & \\
\hline Finger and dummy sucking & 25.0 & 25.0 & \\
\hline Mean weeks KMC & 12.6 & 5.4 & $t=2.14, \mathrm{p}=0.04$ \\
\hline Mean weeks hospitalised & 8.4 & 5.9 & $t=2.72, p=0.01$ \\
\hline Mean mothers' age (years) & 29.6 & 27.7 & $t=0.81, p=0.43$ \\
\hline Mean fathers' age (years) & 34 & 32.5 & $t=0.76, p=0.45$ \\
\hline Parents' marital status, \% & & & $\chi^{2}=3.8, p=0.29$ \\
\hline Married/living together & 83.3 & 58.3 & \\
\hline Single & 16.7 & 41.7 & \\
\hline Mothers' education, \% & & & $\chi^{2}=3.6, p=0.31$ \\
\hline Matric & 50.0 & 58.3 & \\
\hline Other & 50.0 & 41.7 & \\
\hline Fathers' education, \% & & & $\chi^{2}=3.5, p=0.33$ \\
\hline Matric & 58.3 & 75.0 & \\
\hline Other & 41.7 & 25.0 & \\
\hline Mothers' occupation, \% & & & $\chi^{2}=4.2, p=0.12$ \\
\hline Unemployed & 83.3 & 58.3 & \\
\hline Unqualified/casual & 8.3 & 0 & \\
\hline Professional & 8.3 & 41.7 & \\
\hline Fathers' occupation, \% & & & $\chi^{2}=2.1, p=0.36$ \\
\hline Unemployed & 0 & 8.3 & \\
\hline Unqualified/casual & 75.0 & 83.3 & \\
\hline Professional & 25.0 & 8.3 & \\
\hline
\end{tabular}

At pre-test, infants in both groups experienced difficulties in terms of low registration, sensory sensitivity and low thresholds, and for the EG, sensory avoiding behaviour. Eight infants from each group presented within the typical performance range for sensory seeking behaviour.

The numbers of EG infants showing meaningful post-test improvements were as follows: low-registration behaviour $n=5$, sensory sensitive $n=6$, sensory avoiding $n=5$, and low-threshold behaviour $n=6$. In contrast, the numbers of CG infants showing an increase in sensory processing difficulties were as follows: lowregistration behaviour $n=1$, sensory sensitive $n=2$, sensory avoiding $n=7$, and low-threshold behaviour $n=2$. Sensory seeking behaviour improved in $5 \mathrm{EG}$ and $2 \mathrm{CG}$ infants.

\section{Test of Sensory Functions in Infants}

At pre-test, the majority of infants in both groups presented with normal scores in terms of reactivity to tactile deep pressure, visual tactile integration, ocular motor control and reactivity to vestibular stimulation (Fig. 2). Deficient adaptive motor functions were noted in $10 \mathrm{EG}$ and $9 \mathrm{CG}$ infants, who were not able to participate in meaningful and developmentally appropriate ways in reaction to sensory stimuli. ${ }^{[5]}$

EG post-test results showed improvement on all subtests. Numbers of infants showing improvements were as follows: normal reactivity to tactile deep pressure $n=4$, ocular motor control $n=2$, reactivity to vestibular stimulation $n=5$, adaptive motor functions $n=7$, and visual tactile integration $n=4$. The CG post-test results showed similar or poorer performance for all infants in all subtests.

\section{Bayley III Scales of Infant and Toddler Development}

The B-III consists of five subtests (two of which measure two separate components), characterised according to seven levels of performance, ranging from very superior to extremely low. ${ }^{[5]}$

Fig. 3 shows the pre- and post-test distributions on all B-III components for both groups. At pre-test, both groups' mean scaled scores (SSs) for all subtests ranged from average to low average. 


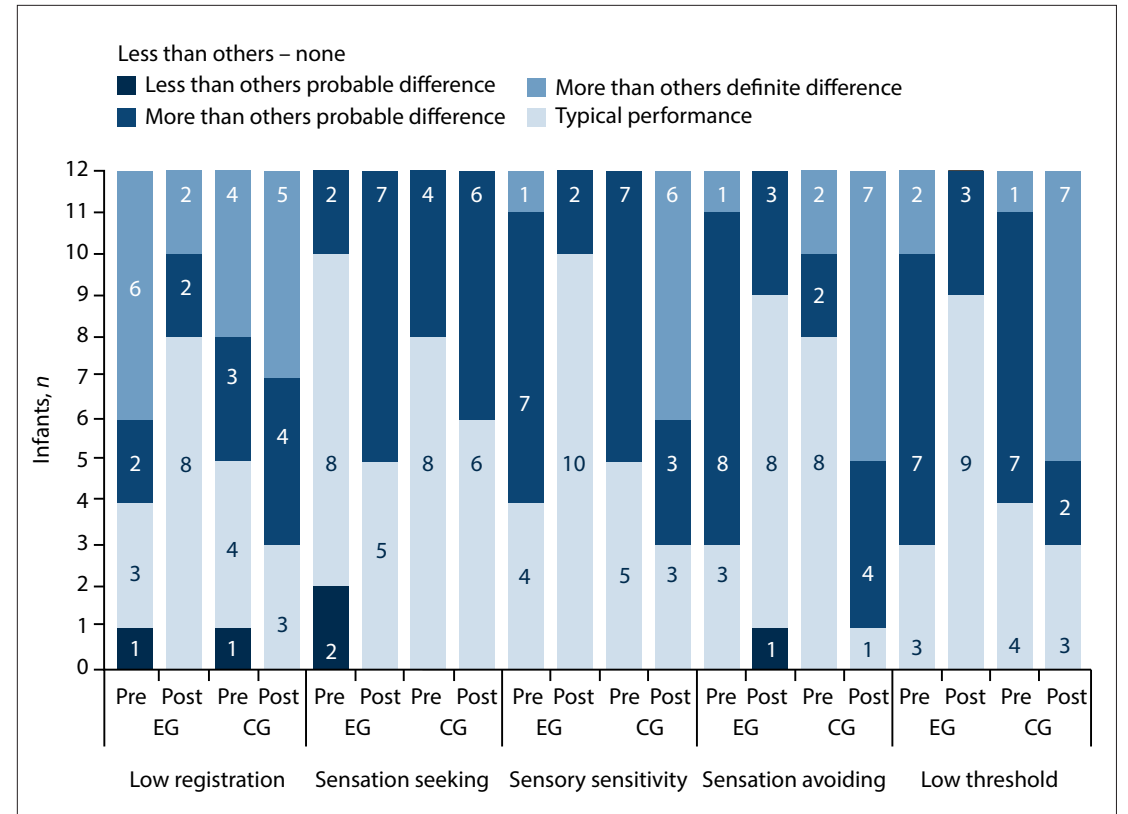

Fig. 1. Infant/Toddler Sensory Profile pre- and post-test results.

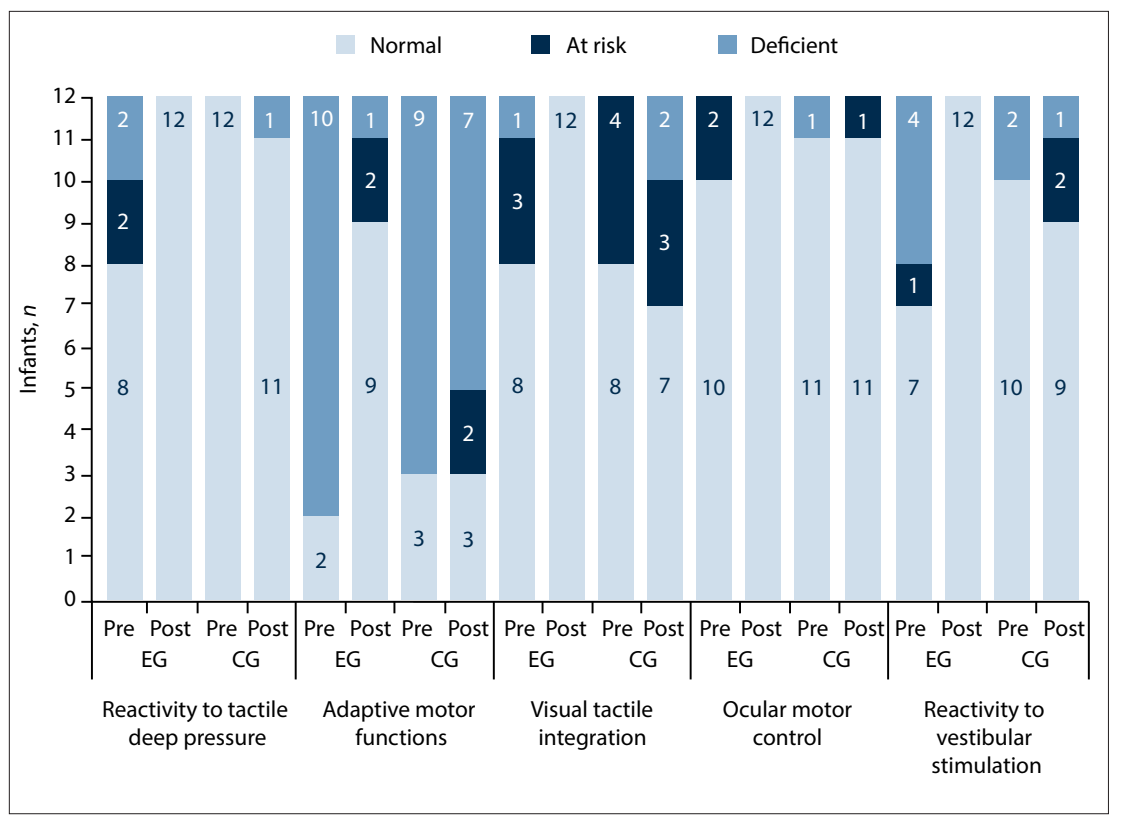

Fig. 2. Test of Sensory Functions in Infants pre- and post-test results.

No statistically significant difference was found between the pre-test results of the two groups on any subtests.

At post-test, Student's $t$-tests for group differences on the level of improvement indicated statistically significant differences between the SSs of the two groups on certain B-III components ( $t$-values, corresponding $p$-values and Cohen's $d$ effect size values are provided for subscale discussions below). Fig. 4 shows the improvement or worsening of each respondent (separated by group) on each B-III component. The EG showed greater improvement than the CG, which had slight improvement or poorer from 7 (average) to 13 (superior). The mean SS for the CG improved slightly from 6.7 to 7.2, but 6 infants still showed SSs from 1 (extremely low) to 7 (low average). All EG infants improved, but 3 CG infants worsened.

Expressive communication $(t=4.25, p=0.08$, $d=1.27)$. The mean SS for the EG improved from 8.1 to 10.7, and post-test SSs ranging between 9 and 12 (high average) indicated a significant improvement. The median of 11 (high average) indicated that $50.0 \%$ of infants had SSs between 11 and 12. The CG had significantly poorer performance levels compared with their pre-tests. The mean SS dropped from 9.0 to 7.5 , and SSs ranged from 4 (borderline) to 10 (high average). Eleven EG infants showed noticeable expressive language improvement, compared with 8 CG infants who showed poorer performances.

Fine motor $(t=2.89, p=0.01, d=0.92)$. The mean post-test SS for the EG of 12 (high average), up from 9 (average), indicated a significant improvement, with SSs ranging from 7 (low average) to 16 (high average). The mean SS for the CG dropped slightly from 9.4 to 9.0 (average). One EG infant showed a significant improvement in fine motor skills, compared with 9 CG infants who showed poorer fine motor skills.

Gross motor ( $t=1.86, p=0.08, d=0.57$ ). Both groups showed improvements, but the EG (mean SS up from 6.0 to 9.4 (average), SSs ranging from 6 (low average) to 15 (very superior)) surpassed the CG (mean SS up from 6.8 to 8.1 (average), SSs ranging from 3 (extremely low) to 14 (superior)). One EG infant showed significant improvement, and only 2 infants' SSs remained the same, compared with 9 CG infants with SS improvement and 3 with poorer gross motor skills.

Social-emotional $(t=0.24, p=0.81, d=0.08)$. Both groups showed improved performance, but the mean SS improvement of 2.7 for the EG slightly exceeded the CG's SS improvement of 2.3. Eleven EG and 8 CG infants showed improved social-emotional skills.

Adaptive behaviour ( $t=1.55, p=0.14, d=0.59$ ). The EG had a mean SS increase of 4.4, compared with a CG SS decrease of 2.2. Nine EG and 6 CG infants showed better adaptive behaviour.

\section{Discussion}

The results are discussed according to levels of modulation, discrimination and refined use (Kramer and Hinojosa). ${ }^{[19]}$

\section{Self-soothing methods}

Adequate modulation of sensory input supports the ability of an infant to sustain opti- 


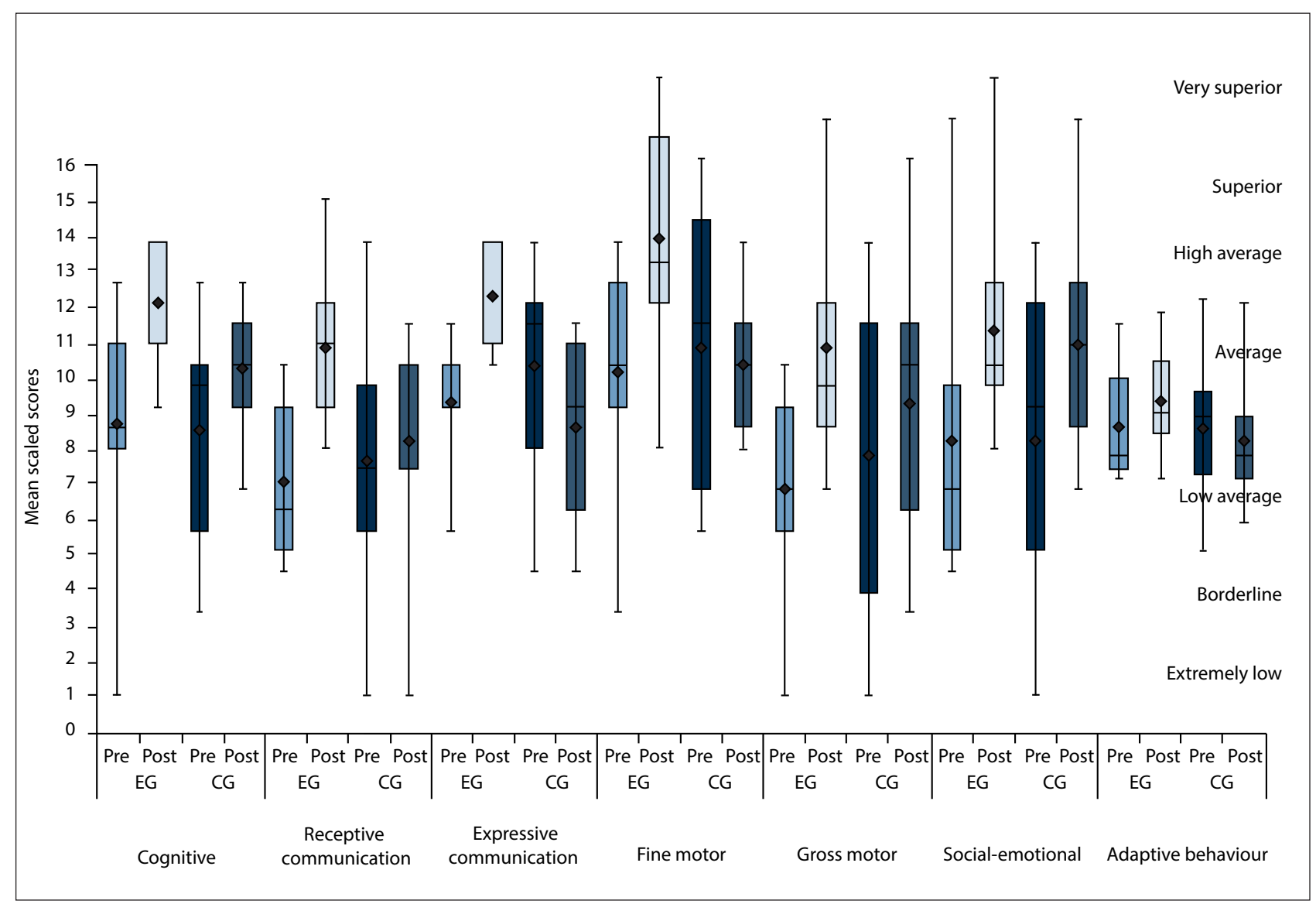

Fig. 3. Results of the Bayley III Scales of Infant and Toddler Development.

mal levels of arousal for engagement in activities, and contributes to stability in emotions and behaviour. ${ }^{[19]}$ Both this and the impact of modulation on an infant's learning and development made it necessary to address self-regulation in terms of intervention goals for inclusion in the interpretation of results for this study.

No statistically significant difference existed between the two groups' use of self-soothing methods at the time of the pre-tests. The effect of training the EG mothers in ways to encourage self-soothing was evident when the EG post-test results indicated that $83.3 \%$ of infants used finger and/or dummy sucking, and all made use of the provided soft taglet. One infant relied on breastfeeding to self-soothe (instead of a finger/dummy) - this was respected as a personal and cultural choice of the mother.

The CG infants tended to use less appropriate self-soothing methods. At post-test, $41.7 \%$ of CG infants used only dummy sucking, $25 \%$ used only finger sucking and $33.3 \%$ used neither. One infant relied on being breastfed to be calmed. The EG had more consistent self-soothing strategies and made use of multiple self-soothing methods.

In order for learning and/or development to take place, sensory stimuli have to be registered by the brain before modulation of the stimuli can support the infant in maintaining optimal levels of arousal to sustain engagement in activities. ${ }^{[14]}$ The premature infant's immature central nervous system after birth and exposure to stressors in the NICU make encouragement of appropriate selfsoothing methods after discharge of the utmost importance in order to help these infants process sensory information. It was concluded that the use of appropriate self-soothing methods by EG infants improved their ability to self-regulate.

It is hypothesised that, as a result of EG parents' involvement in intervention sessions and through taking responsibility for recom- mendations provided, they gained insight into the importance of appropriate self-soothing methods for self-regulation. This could have had a positive effect on the EG's sensory processing in terms of modulation and their ability to engage in learning opportunities.

\section{The Infant/Toddler Sensory Profile}

Results of the ITSP were analysed and compared in terms of behaviours consistent with high and low neurological thresholds. Initially, infants in both groups experienced difficulties in terms of low registration, sensory sensitivity and low thresholds, as well as sensory avoiding behaviour in the EG. Most infants in both groups fell within the typical performance range for sensory seeking behaviour, corresponding to a developmental trend of infants and toddlers aged 7 - 36 months. ${ }^{[7]}$ The post-test results indicated that infants in both groups showed a tendency to be more sensory seeking, especially the EG. Infants in both groups showed an increased interest in their environment - necessary for optimal development.

Infants with developmental delays and sensory processing disorders can experience difficulties in terms of low-registration behaviour and can also have low neurological thresholds for certain stimuli, as seen in the pre-test results. These infants tend to be more sensitive and/or withdraw from stimuli they notice, an indication of poor modulation. ${ }^{[20]}$

The EG post-test results showed significant improvement in terms of infants presenting within the typical performance ranges of low-registration, sensory sensitivity, sensory avoiding and low neurological threshold behaviours. In comparison, the CG showed an increase in sensory processing difficulties in all four quadrants, especially with sensory avoiding behaviour. 


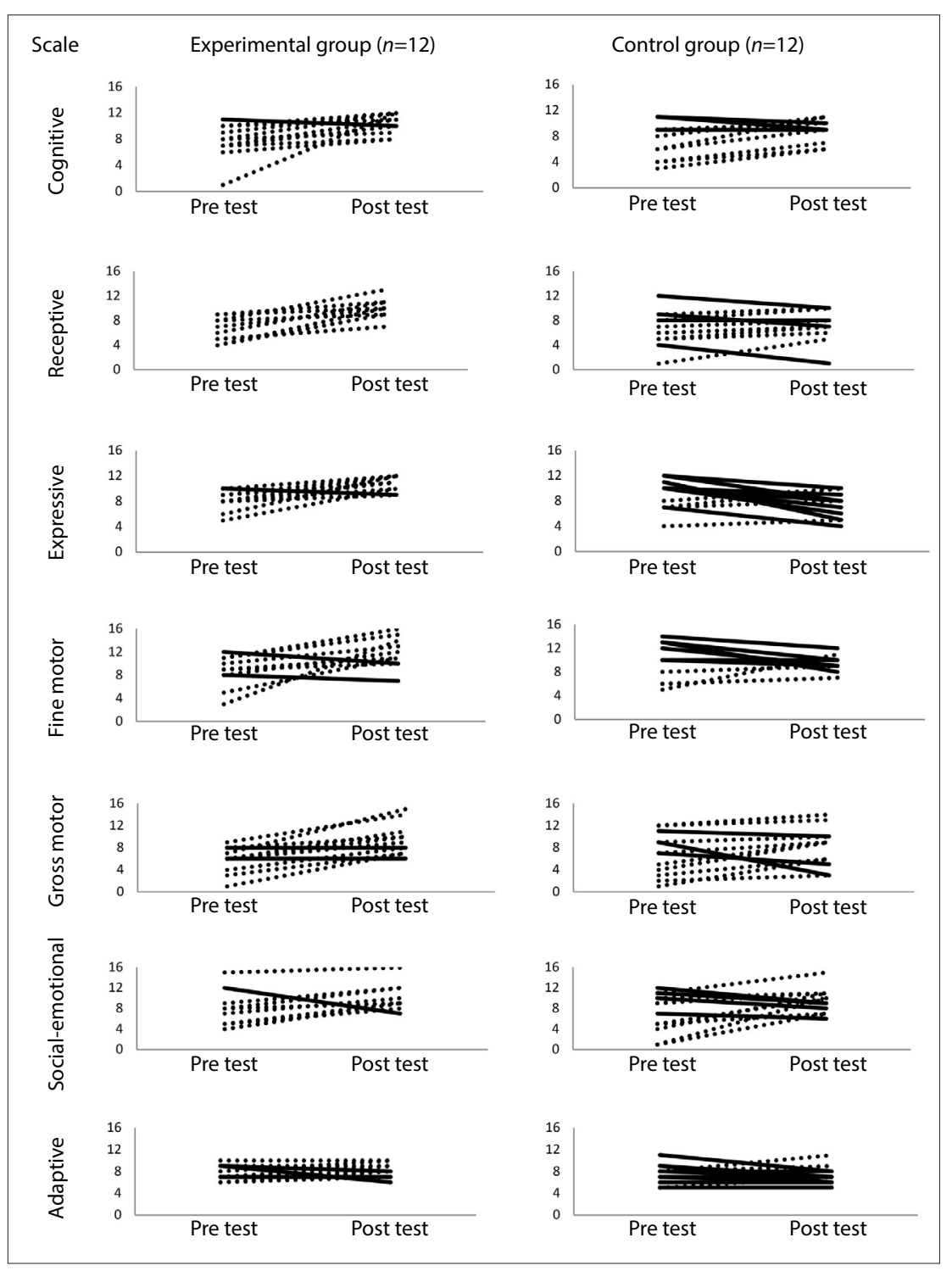

Fig. 4. Pre- to post-test change in the Bayley III Scales of Infant and Toddler Development. Improvement is indicated by the dashed line, and deterioration by the solid line.

Post-test results indicated that EG infants were more tolerant of various sensory stimuli and were more aware of their environment and the possible sensory input it provides, and their behaviour therefore tended towards seeking sensory input.

Test of Sensory Functions in Infants Discrimination within the different sensory systems is about interpreting the qualities of the sensory information and adding meaning to the forming of perceptions. ${ }^{[20]}$ An infant with sensory discrimination difficulties will not know the qualities of the sensory experiences within the sensory system, since the necessary information is not provided by the sensory system. Discrimination difficulties can be found in a single system, but are often seen in a combination of sensory systems, e.g. somatosensory or vestibular-proprioception. ${ }^{[12]}$

The five subdomains of sensory processing and reactivity in infants measured by the TSFI have a strong impact on the development of SI in infants. Pre-test results indicated that a large majority of infants in both groups presented with normal scores in terms of reactivity to tactile deep pressure, visual tactile integration, ocular motor control and reactivity to vestibular stimulation. The majority of the infants were therefore able to register, modulate and discriminate sensory information in their tactile, proprioceptive and vestibular systems.

However, infants in both groups also presented with significant adaptive motor difficulties, confirming that premature infants experience difficulties in terms of discrimination of sensory input for adaptive, functional use. This is concerning, since these infants are not able to participate in meaningful and developmentally appropriate ways in reaction to sensory stimuli from activities of daily living and play. ${ }^{[5]}$

At post-test, EG presented with a marked improvement in terms of all subtests (especially adaptive motor behaviour) compared with the similar or poorer performance in all subtests for the CG. The improvement of the EG infants' adaptive behaviour indicated that exposure to ASI intervention enabled them to participate in meaningful and developmentally appropriate ways in sensory experiences, allowing for optimal learning and functional use.

\section{Bayley III Scales of Infant and Toddler Development}

The subtests of the B-III represent the endproducts of typical SI, ${ }^{[5]}$ including the ability to concentrate, academic learning abilities, capacity for abstract thought and reasoning, organising behaviours, positive self-esteem, self-control and self-confidence, and specialisation of each side of the body and brain.

EG infants improved in all areas of development, with significant improvement in terms of cognitive, receptive and expressive communication, and fine motor, gross motor and social-emotional development. CG infants showed slight improvements in terms of cognitive and receptive communication, and gross motor and socialemotional development. It is reasonable to expect some CG developmental improvement over a period of 10 weeks, due to normal growth and development. However, the developmental improvement in the EG was more significant. Also, CG infants showed an overall decline in expressive communication, and fine motor and adaptive behaviour development.

It is hypothesised that the significant developmental improvement in the EG was the result of these infants' general improvement in sensory modulation abilities. This allowed them to reach the optimal calm-alert state during activities, with improvements in discriminatory abilities that further enabled them to add meaning to what they experienced and to react adaptively.

The authors therefore conclude that ASI intervention had a positive effect on the development of the EG over a period of 10 weeks.

\section{Recommendations}

The low socioeconomic status of the sample strengthened the comparability of the two 
groups and the reliability of the study results. It would be valuable, however, to conduct the same study on premature infants from higher socioeconomic settings, and compare the results of both studies. ASI intervention was shown to have a marked positive effect on premature infants' sensory development, and should be considered as part of the treatment plan for these infants.

\section{Conclusion}

This study indicated that a short period of weekly ASI intervention sessions had a noticeable positive effect on premature infants' sensory processing in terms of registration, modulation and discrimination, contributing to their ability to develop supportive skills for optimal development. Early ASI intervention contributes to enhanced cognitive, language, motor, social-emotional and adaptive motor development, and a lack of ASI intervention contributes to a deterioration of developmental and behavioural outcomes.

Acknowledgements. The authors thank Dr Daleen Struwig, medical writer/editor, Faculty of Health Sciences, University of the Free State (UFS), for technical and editorial preparation of the manuscript; the study participants and their parents who made this study possible; and the South African Institute for Sensory Integration (SAISI).

Author contributions. EL performed the research as part of a master's degree in occupational therapy and wrote the article; AvJ was the study leader and assisted with writing of the article; JR performed the statistical analysis of data and co-authored the article; and $\mathrm{RvH}$ was the co-study leader and co-authored the article. All the authors approved the final version of the article.

Funding. This research was mainly self-funded as part of EL's M (Occupational Therapy) studies. A financial contribution was made by the SAISI as well as the Research Committee of the School for Allied Health Professions of the UFS.

\section{Conflicts of interest. None.}

1. Feldman F, Eidelman AI. Neonatal state organization, neuromaturation, mother-infant interaction, and cognitive development in small-for-gestational-age premature infants. Pediatrics 2006;118(3):e869-e878. https://doi.org/10.1542/peds.2005-2040

2. Lubbe W. Prematurity: Adjusting Your Dream. Pretoria: Little Steps, 2008

3. Abbot A. Neuroscience: The brain, interrupted. Nature 2015;518(7537):24-26. https://doi. org $/ 10.1038 / 518024 a$

4. Lake A. World Economic Forum, Annual Meeting. The first 1,000 days of a child's life are the most important to their development - and our economic success. 2017. https://www.weforum.org/ agenda/2017/01/the-first-1-000-days-of-a-childs-life-are-the-most-important-to-their-development-andour-economic-success/ (accessed 21 May 2017)

5. Ayres AJ. Sensory Integration and the Child: Understanding Hidden Sensory Challenges. 25th anniversary edition. Los Angeles, Calif:: Western Psychological Services, 2008.

6. Parham LD, Cohn ES, Spitzer S, et al. Fidelity in sensory integration intervention research. Am J Occup Ther 2007;61(2):216-227. https://doi.org/10.5014/ajot.61.2.216

7. Dunn W. The Infant/Toddler Sensory Profile Manual. San Antonio, Texas: The Psychological Corporation, 2002.

Williamson GG, Anzalone ME. Sensory Integration and Self-regulation in Infants and Toddlers: Helping Very Young Children Interact with Their Environment. Washington, DC: National Center for Infants, Toddlers and Families, 2001.

9. DeGangi GA. Pediatric Disorders of Regulation in Affect and Behavior: The Therapist's Guide to Assessment and Treatment. San Diego, Calif.: Academic Press, 2000.

0. Schaaf RC, Mailloux Z. Clinician's Guide for Implementing Ayres Sensory Integration: Promoting Participation for Children with Autism. Bethesda, Md.: AOTA Press, 2015

1. Schaaf RC. Smith Roley S. Sensory Integration: Applying Clinical Reasoning to Practice with Diverse Populations. Austin, Texas: Pro-Ed Inc., 2006:275-311.

2. Van Jaarsveld A. Discussion on clinical reasoning on possible difficulties and dysfunctions. South African Institute for Sensory Integration Newsletter 201 1;21(3):7-13.

3. Health24. Preterm birth an African killer. 16 November 2012. http://www.health24.com/Parenting Child/News/Preterm-birth-an-African-killer-20130210 (accessed 2 February 2017)

4. Van Jaarsveld A. Sensory integration and socio-economic environment. South African Institute for Sensory Integration Newsletter 2010;20(2):8-13.

15. Schaaf RC, Anzalone ME. Sensory integration with high-risk infants and young children. In: Smith Roley S, Blanche EI, Schaaf RC, eds. Understanding the Nature of Sensory Integration with Diverse Populations. San Antonio, Texas: Therapy Skill Builders, 2001:275-311.

16. Bayley N. Bayley Scales of Infant and Toddler Development Administration Manual. 3rd ed. San Antonio, Texas: Harcourt Assessment Inc., 2006.

17. DeGangi GA, Greenspan SI. Test of Sensory Functions in Infants (TSFI) Manual. Los Angeles, Calif. Western Psychological Services, 1993.

18. Perlman SB, Pelphrey KA. Regulatory brain development: Balancing emotion and cognition. Soc Neurosci 2010;5(5-6):533-542. https://doi.org/10.1080/17470911003683219

19. Kramer P, Hinojosa J. Frames of Reference for Pediatric Occupational Therapy. Baltimore, Md.: Lippincott Williams \& Wilkins, 2010.

20. Ayres AJ. Developmental Dyspraxia and Adult-onset Apraxia. Torrence, Calif:. Sensory Integration International, 1985

Accepted 29 June 2017 